\title{
VALIDITY OF C- REACTIVE PROTEIN IN DIAGNOSIS OF NEONATAL SEPSIS.
}

\section{Pediatrics}

Ghongade P. G.

Resident (JR III), Department of Pediatrics, Government Medical College, Aurangabad, Maharashtra.

Khaire P. B.*

Professor \& Head, Department of Pediatrics, Government Medical College, Aurangabad,

Maharashtra. *Corresponding Author

\section{ABSTRACT}

Background: Neonatal sepsis with its high incidence \&grave prognosis, in spite of adequate treatment with modern antibiotics, has been a challenge for all times. Optimal diagnosis and treatment strategies are difficult to define. It is essential to diagnose early with laboratory investigation like serial CRP; so that a feasible, rapid and a relatively economic method to diagnose neonatal sepsis at earliest can be instituted even at basic health care level. hence a study was planned to find out the role of CRP against blood culture in early detection of neonatal sepsis. Aim \& Objective: To evaluate Validity of C-Reactive Protein as a screening test in neonatal sepsis. Material and Method: This prospective study was carried out inpaediatric dept of medical college. 100 neonates $(\leq 28$ days $)$ with suspected neonatal sepsis having a birth weight of $\geq 1000$ grams admitted during a period from January 2020 to March 2020 were screened primarily with C-Reactive Protein. Serial level of CRPon the day of admission, $2^{\text {nd }}, 4^{\text {th }}, 6^{\text {th }}, 8^{\text {th }} \& 10^{\text {th }}$ day was compared with the serial blood cultureon the day of admission, $8^{\text {th }}, 15^{\text {th }} \& 21^{\text {st }}$ day to establish the validity of CRP as a screening test. Data analysis carried out by Percentages, Chi Square test, Sensitivity, Specificity, Positive predictive value, Negative predictive value. Results: Amongst 100 neonate $76 \%$ were early neonates, $65 \%$ were low birth weight,CRP was having high sensitivity \& specificity(78.57\%,76.74\% respectively). ROC analysis showed AUC 0.8 with $\mathrm{p}<0.001$.Conclusion: CRP is a good screening test \& establishes its validity in diagnosing suspected sepsis.

\section{KEYWORDS}

Sepsis, C-Reactive protein, Validity.

\section{INTRODUCTION:}

Neonatal Sepsis is defined as a clinical syndrome which occurs in the first 28 days of life which is characterized by symptomatic systemic illness due to infectious agents. ${ }^{2}$ Despite the advances in neonatal care in the recent years, sepsis still remains a life-threatening condition. UNICEF data estimated that 2.8 million neonatal deaths that occurred globally in the year 2013 were due to sepsis and two-thirds of these neonatal deaths have occurred in the developing countries. ${ }^{1}$ The global incidence of neonatal deaths caused by sepsis is only $15 \%$ but in a developing country like India, sepsis accounts for about 30 to $50 \%$ of neonatal mortality.

Neonatal sepsis with its high incidence and its grave prognosis, in spite of adequate treatment with modern antibiotics, has been a challenge for all times. Optimal diagnosis and treatment strategies are difficult to define. The signs and symptoms are protean with a high mortality and thus there is urgent need to know whether the baby has sepsis, to institute treatment as quickly as possible. Confirmation of the diagnosis by definitive culture is not possible rapidly. ${ }^{4}$ The alteration in the immune system of the neonates in comparison to adults like reduced function of the neutrophils, presence of low concentration of immunoglobulin and defects in the complement system both qualitative and quantitative makes them more susceptible to infections. The pathogenic organism after gaining access into the blood stream sometimes causes overwhelming infection without any localisation resulting in sepsis. Clinical suspicion and timely diagnosis isimportant as even a few hours of delay in initiation of treatment results in rapid clinical deterioration of the neonate. The causes of neonatal sepsis may vary from time to time but it is usually of bacterial origin. Based on the onset of symptoms and probable mode of transmission, neonatal sepsis may be of following categories : a) Early onset sepsis. b) Late onset sepsis. c) Very late onset sepsis. ${ }^{1}$ Neonatal sepsis is a clinical syndrome of bacteraemia characterized by systemic signs and symptoms of infection in the first month of life. Neonatal sepsis encompasses systemic infection of the newborn including sepsis, meningitis, pneumonia, arthritis, osteomyelitis and urinary tract infection of the newborn.

Globally of the 130 million babies born every year, about 4 million die in the first 4 weeks of life, i.e. neonatal period. The main direct causes of neonatal deaths are estimated to be preterm birth (28\%), severe infection (26\%), and birth asphyxia (23\%). ${ }^{6}$ According to recent data from National Neonatal Perinatal Database (NNPD) 2002-03 collected from 18 centres from various parts of India, incidence of neonatal sepsis has been reported to be 29.9 per 1000 live births. Early onset sepsis contributed $67 \%$ of all sepsis. Meningitis contributed to
$10.6 \%$ of all cases of sepsis. Neonatal sepsis was one of the common causes of neonatal mortality contributing to $16 \%$ of all intramural deaths.

Diagnosis of neonatal sepsis based on clinical symptoms is not possible. Although isolating its causative microorganisms using blood culture has been the gold standard for its diagnosis, but result will take 24-72 hours after sampling and during this period, it is necessary to treat suspicious infants for sepsis with antibiotics according to clinical symptomsand risk factors. ${ }^{8}$ CRP is synthesized within 6 to 8 hours of exposure to an infective process or tissue damage. It has a half-life of 19 hours and may increase more than 1000-fold during an acute phase response. ${ }^{9}$ The ability to early diagnosis or rule out neonatal sepsis results in to limit inappropriate antibiotic exposure and lowering the cost of therapy. ${ }^{10}$ Blood culture which is considered as gold standard for diagnosis is costly and time consuming (preliminary results are delayed for at least 48 hours or more). Moreover, the yield of blood culture is between $30 \%-70 \%$, hence some neonates with sepsis go undetected.

Various studies have shown that raised CRP had high sensitivity, specificity, positive and negative predictive value for neonatal sepsis. Detection of raised CRP in blood can be done rapidly by cheap and easily available kits. Since India is a developing country with high neonatal morbidity and mortality, there is requirement of rapid and economic diagnostic method to raise our medical health care levels to a next step." Successful treatment thus depends on early clinical diagnosis, vigorous therapy often prior to availability of definite clinical and bacteriological evidence. The wide spread use of antibiotic has not only changed the bacterial pattern responsible for neonatal sepsis but has also brought into the focus the problem of drug resistant bacteria. Hence on account of fulminating nature of the illness and emergence of resistant bacteria, it is essential to diagnose early with laboratory investigation like serial CRP; hence a study was planned to find out the role of CRP against blood culture in early detection of neonatal sepsis, so that a feasible, rapid and a relatively economic method to diagnose neonatal sepsis at earliest can be instituted even at basic health care level.

Objectives: To evaluate Validity of C-Reactive Protein as a screening test in neonatal sepsis.

Material and Method: This prospective observational study was carried out to evaluate Validity of C-Reactive Protein as a diagnostic test in neonatal sepsis. 
Inclusion criteria- All the neonates $(\leq 28$ days) with suspected neonatal sepsis having a birth weight of $\geq 1000$ gramsadmitted during a period from January 2020 to March 2020 included in the study.Suspected neonatal sepsis was considered if neonate had clinico pathological features i.e. maternal pyrexia (within 1 week prenatal and/or 48 hours postnatal), prolonged rupture of membranes (18 hours), foul smelling vaginal discharge and/or maternal urinary tract infection diagnosed in last month of delivery, neonates with $\mathrm{h} / \mathrm{o}$ of birth asphyxia, low birth weight \& prematurity. Neonates having unexplained hypothermia or hyperthermia, lethargy, irritability, poor feeding or milk intolerance, respiratory dysfunction evidenced by apnoea ( $>20 \mathrm{sec}$.), tachypnoea ( $>60$ breaths/minute), cardiovascular dysfunction such as persistent tachycardia ( $>160$ beat $/ \mathrm{min})$ or bradycardia $(<100$ beats/min), poor peripheral circulation, hypotonia or circumoral cyanosis or pallor were also included.

\section{Exclusion criteria-}

Neonates undergone surgery because of risk of wound infection, neonates with congenital anomalies, Prior administration of an Antibiotic regimen for $>24$ hours in neonates \& neonates with any metabolic disorder were excluded from study. Study undertaken by the Department of Paediatrics (Pediatrics ward and NICU) of Government Medical College Aurangabad. Estimated sample size was calculated using standard statistical formula which was standardised with the estimate of prevalence from a previous study. ${ }^{11}$ Estimated prevalence $(\mathrm{P})=6$; Confidence interval $=95 \%(\mathrm{z}=1.96)$; Absolute precision $(\mathrm{d})=5 \% ; \mathrm{n}=\mathrm{z} 2[\mathrm{P}(1-\mathrm{P})] \div \mathrm{d} 2 ; \mathrm{n}=1.96 \times 1.96[0.06(1-0.06$ ) $\div 0.05 \times 0.05$. Using this formula sample size comes out to be 92 considering the admission rate, inflow of the cases \& loss to follow up at our tertiary care centre. 100 neonates were finally enrolled. Neonates of parents who were willing to give written informed consent and fulfilling above inclusion \& exclusion criteria were assessed clinically with proper history, clinical examination and investigations as per performa

All the neonates who were clinically symptomatic / suspected to have sepsis were screened primarily using CRP and at the same time blood culture was also sent. On admission before giving antibiotic, under all aseptic precaution $2 \mathrm{ml}$ of blood was drawn and inoculated into a BHI broth (BRAIN HEART INFUSION BROTH) \& sent to microbiology lab. Serial blood culture were evaluated on the day of admission, $8^{\text {th }}$ day, $15^{\text {th }}$ day $\& 21^{\text {st }}$ day. CRP done by latex slide agglutination test and consider as positive $\geq 6 \mathrm{ug} / \mathrm{lit}$ ( $6 \mathrm{micro}$ gram $/ \mathrm{ml}$ ). Serial level of CRP were evaluated on the day of admission, $2^{\text {nd }}, 4^{\text {th }}, 6^{\text {th }}, 8^{\text {th }} \& 10^{\text {th }}$ day. Analysis was carried out on admission before treatment $\left(0^{\text {th }}\right.$ day CRP \& $0^{\text {th }}$ day blood culture $) \&$ after treatment $\left(10^{\text {th }}\right.$ day CRP $\& 8^{\text {th }}$ day blood culture) to evaluate serial monitoring of CRP against blood culture. Relevant to clinical situation CSF, Urine analysis and swabs of infective focus were taken \& other routine investigations carried out. The empirical antibiotic therapy was started according to antibiotic guidelines in the NICU, if CRP was positive, awaiting the blood culture reports.Simultaneously Pilot testing was done to establish validity of performa.Data analysis carried out by Percentages, Chi Square test, Sensitivity, Specificity, Positive predictive value, Negative predictive value. The "p" value of less than 0.05 accepted as an indicator of statistical significance. Data analysis carried out using Microsoft MS Excel \& Epi Info statistical software.

Approval from the IEC / IRB (Institutional Ethical Committee Institutional Review Board) was taken.

\section{RESULTS :}

The number of of neonates participated in the study were 100 . Out of those neonates $76 \%$ of neonates were early neonates. Male to female ratio was $1 / 1.04$ with slight female preponderance males were $49 \%$, while female neonates were $51 \%$. $65 \%$ neonates were below normal weight with $35 \%$ of the neonates being low birth weight, while $20 \%$ of the neonates having very low birth weight. $62 \%$ of the neonates were full term. $48 \%$ of the neonate were intramurally born. $78 \%$ were born normally while $19 \%$ delivered through lower segment caesarean section. $4 \%$ neonates were having $\mathrm{h} / \mathrm{o}$ of PROM, $2 \%$ Foul smelling liquor, $4 \%$ maternal fever but maximum number of neonates i.e $90 \%$ were having a history of other risk factors for sepsis such as prematurity, LBW, birth asphyxia h/o of prolonged labor etc. (Table no 1)
Table 1: Demographic Characteristics of neonates enrolled in this study

\begin{tabular}{|c|c|c|c|c|}
\hline \multirow{3}{*}{\begin{tabular}{l|} 
Sr. No \\
1
\end{tabular}} & \multicolumn{2}{|c|}{\begin{tabular}{|l|} 
Characteristics \\
\end{tabular}} & \multirow{2}{*}{\begin{tabular}{|l|} 
Frequency \\
76 \\
\end{tabular}} & \multirow{2}{*}{\begin{tabular}{|l|} 
Percentage \\
76 \\
\end{tabular}} \\
\hline & Age & Early neonate & & \\
\hline & & Late neonate & 24 & 24 \\
\hline \multirow[t]{2}{*}{2} & \multirow[t]{2}{*}{ Sex } & Male & 49 & 49 \\
\hline & & Female & 51 & 51 \\
\hline \multirow[t]{3}{*}{3} & \multirow{3}{*}{$\begin{array}{l}\text { Birth } \\
\text { weight }\end{array}$} & Normal & 35 & 35 \\
\hline & & LBW & 45 & 45 \\
\hline & & VLBW & 20 & 20 \\
\hline \multirow[t]{3}{*}{4} & \multirow[t]{3}{*}{ Maturity } & Preterm & 36 & 36 \\
\hline & & Term & 62 & 62 \\
\hline & & Post term & 02 & 02 \\
\hline \multirow[t]{2}{*}{5} & \multirow{2}{*}{$\begin{array}{l}\text { Birth } \\
\text { Place }\end{array}$} & Intramural & 48 & 48 \\
\hline & & Extramural & 52 & 52 \\
\hline \multirow[t]{3}{*}{6} & \multirow{3}{*}{$\begin{array}{l}\text { Mode of } \\
\text { delivery }\end{array}$} & Normal & 78 & 78 \\
\hline & & Assisted & 3 & 3 \\
\hline & & LSCS & 19 & 19 \\
\hline \multirow[t]{4}{*}{7} & \multirow{4}{*}{$\begin{array}{l}\text { Risk } \\
\text { factors }\end{array}$} & H/o PROM & 4 & 4 \\
\hline & & H/o Foul smelling liquor & 2 & 2 \\
\hline & & H/o Maternal fever & 4 & 4 \\
\hline & & H/o others & 90 & 90 \\
\hline
\end{tabular}

An association between blood culture \& CRP levels on the day of admission shows 46 cases showing CRP as well as blood culture positive while 19 cases were showing CRP as well as blood culture as negative. Chi square test applied shows that the difference is significant $(\mathrm{P}<0.017)$ giving a strong association in the CRP levels and blood culture. While the Sensitivity 76.66 , Specificity 47.55 , Positive predictive value 68.65 , Negative predictive value 57.57 . were as follows on the day of admission before treatment. An association between blood culture 8th day \& CRP levels on 10th day in neonates shows 11 cases showing CRP as well as blood culture positive while 66 cases were showing CRP as well as blood culture as negative. Chi square test applied shows that the difference is highly significant $(\mathrm{P}<$ 0.001 ) giving a strong association in the CRP levels and blood culture after treatment when monitored serially. As there is decrease in CRP levels in previously positive cases so also there is concurrent decrease in positive blood culture in neonates as compared to the time of admission. This gives a serial temporal correlation between CRP levels $\&$ blood culture. While the Sensitivity 78.57, Specificity 76.74 , Positive predictive value 35.48 , Negative predictive value 95.65 were as follows after treatment when monitored serially. (Table 2) (Fig 1)

Table 2 : Blood Culture \& CRP Levels at Admission before treatment \& after treatment

\begin{tabular}{|c|c|c|c|c|c|c|}
\hline \multicolumn{7}{|c|}{ At admission } \\
\hline & & \multicolumn{2}{|c|}{ Blood Culture } & \multirow[t]{2}{*}{ Total } & \multirow[t]{2}{*}{ Chi sq } & \multirow{2}{*}{$\begin{array}{l}\mathrm{P}- \\
\text { value }\end{array}$} \\
\hline & & Positive & Negative & & & \\
\hline \multirow[t]{2}{*}{ CRP- Level } & Positive & 46 & 21 & 67 & \multirow[t]{3}{*}{6.3} & \multirow[t]{3}{*}{0.017} \\
\hline & Negative & 14 & 19 & 33 & & \\
\hline \multicolumn{2}{|l|}{ Total } & 60 & 40 & 100 & & \\
\hline \multicolumn{7}{|c|}{ After treatment } \\
\hline & & \multicolumn{2}{|c|}{ Blood Culture } & \multirow[t]{2}{*}{ Total } & \multirow[t]{2}{*}{ Chi sq } & \multirow{2}{*}{\begin{tabular}{|l|} 
P- \\
value
\end{tabular}} \\
\hline & & Positive & Negative & & & \\
\hline \multirow[t]{2}{*}{ CRP-Level } & Positive & 11 & 20 & 31 & \multirow[t]{3}{*}{17.22} & \multirow{3}{*}{0.001} \\
\hline & Negative & 03 & 66 & 69 & & \\
\hline Total & & 14 & 86 & 100 & & \\
\hline
\end{tabular}

Figure 1 : Blood Culture \& CRP Levels at admission before treatment \& after treatment
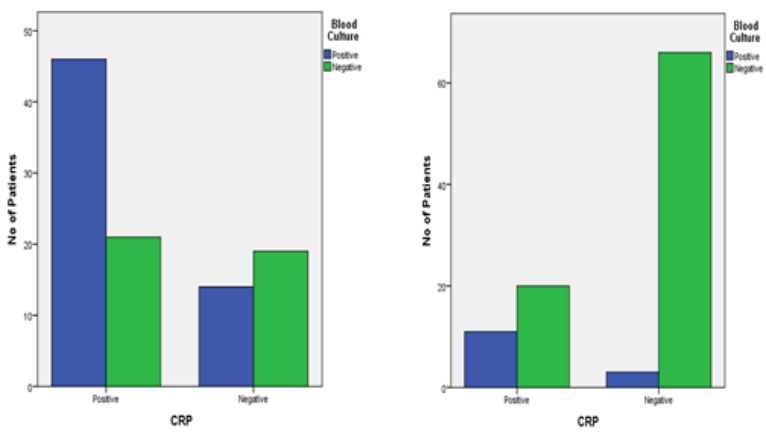

At admission 
Figure no 2 Composite Bar diagram showing serial CRP levels with respect to blood culture. Sensitivity, specificity, PPV \&NPV of $76.66 \%, 47.55 \%, 68.65 \%$ \& $57.57 \%$ respectively on the day of admission (0thday)(from Table no 2) while sensitivity, specificity, PPV \&NPV of $78.57 \%, 76.74 \%, 35.48 \%$ \& $95.65 \%$ respectively on the (10th day)(Table no 3). When we monitor serial levels, the sensitivity \& specificity increases. This establishes diagnostic accuracy (reliability \& validity) of serial level of CRP against blood culture

Figure 2 : Sensitivity, Specificity, Positive Predictive Value (PPV), Negative Predictive Value (NPV) of serial CRP levels with respect to blood culture

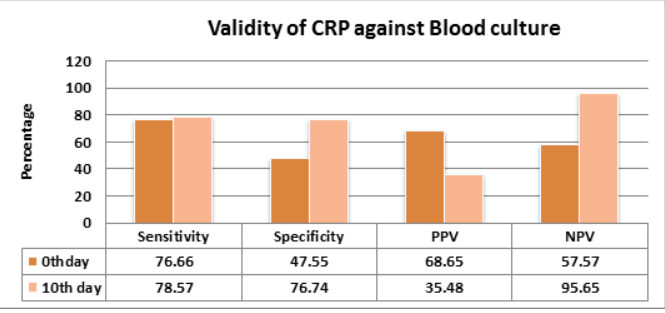

\section{Serial CRP leve}

C- Reactive Protein was found to have a good discriminatory power as a screening test to predict sepsis in neonates on receiver operating characteristic (ROC) analysis. It was compared against the blood culture which is a gold standard confirmatory test. Serial monitoring of CRP with blood culture was carried out \&comparison was made by plotting ROC curve at admission i.e before institution of antibiotic (0th day CRP with 0th day blood culture) $\&$ after treatment i.e (10th day CRP with 8th day blood culture). Area under the curve (AUC) was higher when monitored serially i.e after treatment(AUC : 0.851 ; P value $<0.001$ ) as compared to at admission(AUC : 0.743 ; $\mathrm{P}$ value $<$ 0.001 ). In addition there was significant increase in specificity after treatment while sensitivity was same. Specificity (at admission 47.55; after treatment 76.74). (Table3)(Fig 4)

Table 3 :- Sensitivity \& Specificity of C-Reactive Protein to predict sepsis by Receiver Operating Characteristic Analysis.

\begin{tabular}{|c|c|c|c|c|c|c|c|}
\hline \multirow[t]{2}{*}{ Variable } & \multirow[t]{2}{*}{ Sensitivity } & \multirow[t]{2}{*}{ Specificity } & \multirow[t]{2}{*}{\begin{tabular}{|l|} 
Std. \\
Error
\end{tabular}} & \multirow[t]{2}{*}{\begin{tabular}{|l|} 
P- \\
value.
\end{tabular}} & \multirow[t]{2}{*}{$\begin{array}{l}\text { Area } \\
\text { Under } \\
\text { Curve }\end{array}$} & \multicolumn{2}{|c|}{\begin{tabular}{|l|}
$95 \%$ \\
Confidence \\
Interval
\end{tabular}} \\
\hline & & & & & & $\begin{array}{l}\text { Lower } \\
\text { Bound }\end{array}$ & $\begin{array}{l}\text { Upper } \\
\text { Bound }\end{array}$ \\
\hline \begin{tabular}{|l|} 
CRP At \\
admission
\end{tabular} & 78.66 & 47.55 & 0.050 & 0.001 & 0.743 & 0.645 & 0.841 \\
\hline $\begin{array}{l}\text { CRP After } \\
\text { Treatment }\end{array}$ & 78.57 & 76.74 & 0.045 & 0.001 & 0.851 & 0.762 & 0.940 \\
\hline
\end{tabular}

Figure 3 :- Receiver operating characteristic analysis Curve for CRPat admission\& after treatment

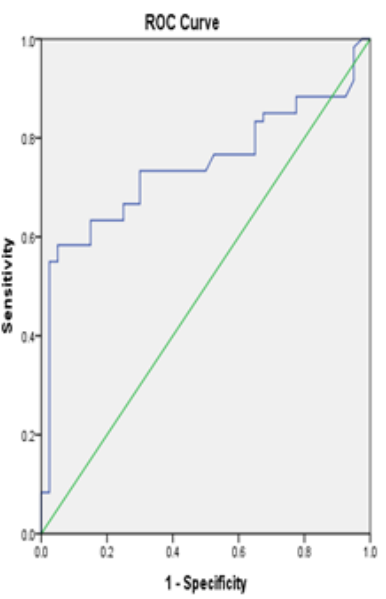

Diagnal segments are ponduces by fiss

At admission

There were various micro organisms isolated from the positive blood cultures. 16 cultures were positive for Kleibsiella pneumoniae, 15 for
Staphylococcus Aureus, 5 for Pseudomonas Aeroginosa, 10 for Gram negative non fermenting rods, while 14 for Actinobacter bowmanii. 40 blood cultures were sterile.

\section{DISCUSSION :-}

In the present study $60 \%$ of the neonates with clinical suspicionof sepsis were found to be culture positive. These findings are in congruence with study done by Mary et al 41 ,Khatua et al, Joshi etal ${ }^{(1,14,15)}$ while other studies showed a less incidence like St Geme et al, Tallur et al ${ }^{(16,17)}$ This wide range in incidence of culture positive neonatal sepsis reported worldwide could be as a result of lack of standard definition of clinical sepsis across different centre's \& lack of proper information about antibiotics received prior to blood culture and difference in culture technique.The male to female ratio of study population was 1:1.04. There is a slight female preponderance for culture positivity in the present study which is against finding of the studies by St geme et al,Khatua et al,Tallur et al \& Kuruvilla et al ${ }^{(16,4,4,1,18)}$

Culture proven sepsis was more common among the preterm and low birth weight babies. Similar findings were reported in various studies including May et al, Khatua et al, Joshi et $\mathrm{al}^{(13,14,15)} \mathrm{LBW}$ and preterm neonates are at high risk of developing sepsis in comparison to a term neonate as they are vulnerable to sepsis due to their intrinsic susceptibility to infection owning to the immature immune system in addition to other factors like prolonged hospital stay, total parenteral nutrition and exposure to invasive procedures. Preterm neonates are more susceptible to infections due to lack of inherent defensive mechanism, both humeral and cellular defense mechanisms. The results of the present study are comparable with the other studies. It is also evident from present study that nearly a high proportion of cases had birth weight $<2.5 \mathrm{kgs}$ and low proportion of Gestational age $<37$ wks as risk factors for developing septicaemia. This is comparable with studies conducted by May et al, Kuruvella et al ${ }^{(13,18)}$ Clinical chorioamnionitis diagnosed by presence of foul smelling liquor \& maternal fever was present in $2 \%$ \& $4 \%$ of the culture proven sepsis in present study which is comparable with studies conducted by St.Geme et al, Takker et al ${ }^{(15,17) \text {. }}$

Further in this study a significant proportion of culture provensepsis was found to be associated with Normal vaginal delivery which is against the findings seen in other studies which showed assisted vaginal delivery using instrumental procedures a susceptibility factor. According to Tallur et al ${ }^{(17)} \mathrm{NVD}$ showed higher rates of culture isolation than the neonates delivered by LSCS which coincides with our study. The foetus is at risk of exposure to the colonized microbial florain the maternal genital tract during labour. The encounter with thepathogens during delivery in contrast to the sterile in-utero environmentaccounts for this high degree of culture positivity among the neonatesdelivered by AVD/NVD than LSCS.

There were $48 \%$ of neonates were intramural admissions. This finding goes against studies done by the report of National Neonatal Perinatal Database 2002- 2003. ${ }^{(7)}$ This probably reflects that Hospital, being a tertiary referral centre for both Obstetrics and Pediatrics cases, has maximum late referral and intervened cases with higher proportion of babies born with adverse intrapartum and neonatal risk factors for neonatal sepsis. The variations in the occurrence of perinatal risk factors probably reflect differences in the rates of occurrence of the predisposing risk factors in the various studies.

The present study was undertaken to assess the utility of CRP test as marker for diagnosis of neonatal septicaemia. Serum concentration of CRP increase several hundred-fold in response to bacterial infection, making it an attractive diagnostic test for neonatal sepsis. The relatively low cost of the readily available CRP test makes it an attractive single test to screen for neonatal sepsis. We got a significant 46 of culture positive septic neonates were CRP positive giving a high sensitivity $76.66 \%$. We found that 19 neonates with culture negative was also CRP negative has a moderate specificity of $47.55 \%$. Similar studies done throughout the world have given a wide range of specificities. a positive predictable value for CRP of $68.65 \%$. \&a negative predictable value for CRP of $57.57 \%$.These findings were similar to studies by Sumit Bhatia et al, R.S Jaiswal et al ${ }^{(11,19)}$ while these findings were against studies such as William E Beniz et al ${ }^{(60)}$ Different studies have used different methods and different cut off value to denote CRP positivity. We used rapid slide latex agglutination qualitative method for determination of CRP and used a cut off value of $6 \mu \mathrm{g} / \mathrm{ml}$ for CRP positivity. The difference in various studies is due to 
different cut-off values used in the qualitative test (kit). We had higher sensitivity because our cut-off was $6 \mathrm{ug} / \mathrm{ml}$. The differences in the results of this parameter shown by the different studies may be due to variations in the blood sampling time, the severity of infection, the age of the neonates.

The sensitivity of CRP is negated during the initial 48 hours of infection since there is only a slow rise. Further elevated CRP concentrations in conditions other than sepsis like PROM and meconium aspiration are found to affect its specificity as well. Hence CRP can be used as a sensitive tool in diagnosing, following the course of treatment and predicting the prognosis in neonatal sepsis. Also it assists in diagnosing sepsis early on the day of admission itself and also prevents inadvertent use of antibiotics thereby reducing further emergence of drug resistant strains.

\section{CONCLUSION :-}

C- Reactive Protein was found to have a good discriminatory power to predict neonates suspected to be having sepsis, so that early institution of antibiotic can be started before waiting for the confirmatory gold standard blood culture results to come. It will ultimately increase the survival rate. Hence CRP is a good screening test \& establishes its validity in diagnosing suspected sepsis.But CRP still cannot be relied as a sole marker. A much detailed intervention and multiple large scale cross sectional studies with a much higher sample size should be performed to further confirm these findings to make serum CRP "the diagnostic marker" of sepsis.

\section{REFERENCES :}

Infections of the Neonatal Infant, Nelson Textbook of Paediatrics; Elsevier publication;Canada; 19thedition; $1 ;$ p 118, 629-648

2. John P. Cloherty, Eric C. Eichenwald, Ann R. Stark. Manual of neonatalcare Philidelphia: Lippincott, Williams and Wilkins Publication;(2012) 7thedition ;p 230232

3. Shahsanam G, Zahra F, Mohammed K., Behrooz I, Farzin A, Hashem Mand Amir H, Coagulase negative Staphylococcus; the most common causeof neonatal sepsis in Urmia, Iran, Iran J Pediatr. (2008); 18,p237-2433.

4. S. Datta, JK Oberoi, TD Chugh. Laboratory diagnosis of Neonatal sepsisJournal S. Dant, JK Ob6;20

5. Rajiv Aggarwal, Nupur Sarkar, Ashok K. Deorari, Vinod K. Paul. Sepsis inthe Newborn. Indian J Pediatr 2001; 68 (12): p1143-7.

6. Lawn JE, Cousens S, Zupan J;Lancet Neonatal Survival Steering Team.4million neonatal deaths: When? Where? Why? Lancet 2005;365(9462):p891-900

7. National Neonatal Perinatal Database. Report 2002-03. Published byNNPD nodal centre, Department of Pediatrics, All India Institute of Medical Science, New Delhi.(2002);1;p125,126.

8. Rassol AL- Musawi KH, Kalaf DK, Karim LA (2013) The bacterial profileand Creactive protein of suspected septic neonates admitted to the Al-Kadyemia teaching reactive protein of suspected septic neonates admitted to the Al-Kadyemia teaching
hospital. Int J Recent Sci Res 4:p1723-1727.

9. Ng PC.(2004) Diagnostic markers of infection in neonates. Arch Dis ChildFetal Neonatal 2004;89:p229-235.66

10. Bang AT, Bang RA, Baitule SB, Reddy HM, Deshmukh MD. (1999)Effect of home based neonatal care and management of sepsis on neonatalmortality: field trial in rural India. Lancet1999;354:p1955-1961.

11. Sumit Bhatia, Chaman R, Verma, Balvir S, Tomar, Bhagwan S, Natani,Pardeep Goyal, Ankit Agarwal. Correlation of CRP and Blood Culture inevaluation of Neonatal Sepsis. Indian Journal of Basic and Applied MedicalResearch; December 2016: Vol.-6, Issue1,p 663-670.

12. Hisamuddin E, Hisam A, Wahid S, Raza G. Validity of C-reactive protein (CRP) for diagnosis of neonatal sepsis. Pak J Med Sci 2015;31(3):p527-531.

13. May M, Daley AJ,Donath S et al. Early onset neonatal meningitis in Australia and New Zealand, 1992 -2002. Arch Dis Child Fetal Neonatal $2005 ; 90$ :p,324-327

14. Khatua SP, Das AK, Chatterjee BD, Khatua S, Ghose B, Saha A. Neonatalsepticemia. Indian J Pediatr 1986; 53 (4):p 509-14.

15. Joshi SG, Ghole VS, Niphadkar KB. Neonatal Gram-Negative Bacteremia. Indian JPediatr 2000; 67(1): p27-32.

16. Joseph W. St. Geme Jr, Dennis L. Murray, JoAnne Carter, Calvin J. Hobel,Rosemary D. Leake, Bascom F. Anthony et al. Perinatal bacterial infectionafter prolonged rupture of amniotic membranes: An analysis of risk andmanagement. J Pediatr 1984 Apr; 104 (4):p, 608-13

17. Tallur SS, Kasturi AV, Shobha D. Nadgir, Krishna BVS. Clinico-bacteriologicai Study of Neonatal Septicemia in Hubli. Indian J Pediatr 2000; 67 (3): p, 169- 74.

18. Kurien Anil Kuruvilla, Swati Pillai, Mary Jesudason, Atanu Kumar Jana. Bacterial profile of Sepsis in a Neonatal unit in South India. Indian Pediatrics 1998 Sept; 35: p, $851-8$

19. Jaswal RS, Kaushal RK, Asha Goel, Kushla Pathania. Role of C-Reactive Protein in Deciding Duration of Antibiotic Therapy in Neonatal Septicemia. Indian Pediatrics 2003 Sept; 40:p, 880-3.

20. Benitz WE, Michael YH, Ashima Madan, Pramela R .Serial serum C-Reactive Protein in the diagnosis of neonatal infection. 1998;102:p, $1-10$ 